\title{
Simulation based Performance Comparison of AODV, FSR and ZRP Routing Protocols in MANET
}

\author{
Ashish K. Maurya \\ Department of Electronics and Computer Engineering \\ Indian Institute of Technology Roorkee \\ Roorkee, India
}

\author{
Dinesh Singh \\ Department of Electronics and Computer Engineering \\ Indian Institute of Technology Roorkee \\ Roorkee, India
}

\begin{abstract}
Mobile Ad hoc NETwork (MANET) is a collection of mobile nodes that are arbitrarily located so that the interconnections between nodes are dynamically changing. In MANET mobile nodes forms a temporary network without the use of any existing network infrastructure or centralized administration. A routing protocol is used to find routes between mobile nodes to facilitate communication within the network. The main goal of such an ad hoc network routing protocol is to establish correct and efficient route between a pair of mobile nodes so that messages delivered within the active route timeout interval. Route should be discovered and maintained with a minimum of overhead and bandwidth consumption. This paper presents performance evaluation of three different routing protocols i.e. Ad hoc OnDemand Distance Vector (AODV), Fisheye State Routing (FSR) and Zone Routing Protocol (ZRP) in variable pause times and variable number of nodes. We have used random waypoint mobility model to design the network and performed simulations by using QualNet version 5.0 Simulator [1] from Scalable Networks. Performance of AODV, FSR and ZRP is evaluated based on Average end-to-end delay, Packet delivery ratio, Throughput and Average Jitter.
\end{abstract}

\section{Keywords}

MANET, AODV, FSR, ZRP, QualNet version 5.0.

\section{INTRODUCTION}

Mobile Ad Hoc Networks are the self-organizing and selfconfiguring wireless networks which do not rely on a fixed infrastructure and has the capability of rapid deployment in response to application needs. Nodes of these networks function as routers which discover and maintain routes to other nodes in the network. The Ad hoc network applications include military applications, casual conferences, meeting, virtual classrooms, emergency search-and-rescue operations, disaster relief operation, automated battlefield and operations in environments where construction of infrastructure is difficult or expensive. In MANET, due to lack of centralized entity and mobile nature of nodes, network topology changes frequently and unpredictably. Hence the routing protocols for ad hoc wireless networks have to adapt quickly to the frequent and unpredictable changes of topology [2]. There are many routing protocols available for Adhoc networks as AODV, CGSR, DSDV, DSR, DYMO, FSR, GSR, OLSR, STAR, TORA, WRP and ZRP etc. In this paper we have used three routing protocols: AODV, FSR and ZRP and evaluated the performance of these three routing protocol as a function of pause time and number of nodes.

\subsection{AODV}

The Ad hoc On Demand Distance Vector (AODV) [3] routing algorithm is a reactive routing protocol designed for ad hoc mobile networks. It builds routes between nodes only as desired by source nodes for transmitting data packets; therefore, it is also known as source initiated routing protocol. It avoids the counting-to-infinity problem by using sequence numbers on route updates such that ensuring the freshness of routes [4]. AODV basically involves two processes, one is route discovery and other is route maintenance. It discovers routes by route request (RREQ) and route reply (RREP) messages and route maintenance is done by HELLO messages and route error (RERR) messages. Each node maintains a route table in which next hop routing information for destination nodes is stored. When a source node wants to know the route to a destination for which it does not already have a route, source node initiates route discovery and broadcasts a route request (RREQ) message across the network. Each node updates the information regarding source node after receiving this RREQ message. When a node rebroadcasts a route request, it sets up a reverse path pointing towards the source node in the route tables (AODV assumes that the links are symmetric and bi-directional). The RREQ message contains source node's IP address, destination node's IP address, current sequence number and broadcast ID, it also contains the last known (most recent) sequence number for the destination of which the source node is aware. A route is created to the source node when RREQ message is received at each intermediate node. If the receiving node is not the destination node and does not have a current route to the destination, it re-broadcast this RREQ message. A node receiving the RREQ may send a route reply (RREP) if it is either the destination or if it has a route to the destination with corresponding sequence number greater than or equal to that contained in the RREQ. In this case, it unicasts a RREP back to the source in a hop-by-hop fashion. Each node records the route request's source IP address and broadcast ID. If any node receives already processed RREQ, nodes discard the RREQ and do not forward it. Intermediate nodes that forward the RREP, also record the next hop to destination. Forward links to the destination are setup when RREP travels along the reverse path to the source. The source node records the route to the destination and can begin forward data messages to the destination node when it receives the RREP. Here the sending of data messages to the destination node is delayed due to route discovery process. If the source node later receives a RREP containing a greater sequence number or contains the same sequence number with a smaller hop-count, it may update its routing information for that destination node and begin using the 
better route. As long as the route remains active, it will continue to be maintained. A route is active if there are data packets periodically travelling from the source node to the destination node along that path. To know about active neighbors each neighboring nodes periodically exchange HELLO messages. When a link break occurs while the route is active, all active neighbors are informed and link failures are propagated by means of route error (RERR) messages to the source node, which also update destination sequence numbers. After receiving the RERR message, the source node invalidates the route and can reinitiate route discovery, if desired.

\subsection{FSR}

Fisheye State Routing (FSR) [5] protocol is a proactive (table driven) ad hoc routing protocol and its mechanisms are based on the Link State Routing protocol used in wired networks. FSR is an implicit hierarchical routing protocol. It reduces the routing update overhead in large networks by using a fisheye technique. Fish eye has the ability to see objects the better when they are nearer to its focal point that means each node maintains accurate information about near nodes and not so accurate about far-away nodes. The scope of fisheye is defined as the set of nodes that can be reached within a given number of hops. The number of levels and the radius of each scope will depend on the size of the network. Entries corresponding to nodes within the smaller scope are propagated to the neighbors with the highest frequency and the exchanges in smaller scopes are more frequent than in larger. That makes the topology information about near nodes more precise than the information about farther nodes. FSR minimized the consumed bandwidth as the link state update packets that are exchanged only among neighboring nodes and it manages to reduce the message size of the topology information due to removal of topology information concerned far-away nodes. Even if a node doesn't have accurate information about far away nodes, the packets will be routed correctly because the route information becomes more and more accurate as the packet gets closer to the destination. This means that FSR scales well to large mobile ad hoc networks as the overhead is controlled and supports high rates of mobility.

The FSR concept originates from Global State Routing (GSR). GSR can be viewed as a special case of FSR, in which there is only one fisheye scope level and the radius is infinite. As a result, the entire topology table is exchanged among neighbors. Clearly, this consumes a considerable amount of bandwidth when network size becomes large.

\subsection{ZRP}

Zone Routing Protocol (ZRP) [8] is a hybrid protocol which combines the advantages of both proactive and reactive approaches. For route discovery reactive routing protocols involves long route request delays and inefficient flooding, while proactive routing protocols uses excess bandwidth to maintain routing information. ZRP [9]-[11] solves these problems by combining the best properties of both approaches. It takes advantage of proactive discovery within a node's local neighborhood, and using a reactive protocol for communication between these neighborhoods. In Ad-Hoc mobile network, it can be supposed that the most communication takes place between nodes closer to each other. Therefore, ZRP reduces the proactive scope to a zone centered on each node. In a limited zone, the routing information can be maintained easily and the amount of routing information that is never used is also minimized. Since all nodes proactively store local routing information, nodes farther away can be reached with reactive routing [8].

The Zone Routing Protocol is based on the concept of zones in which each node may be within multiple overlapping zones, and each zone may be of a different size. The size of routing zone is defined by radius $r$ which expressed in hops. Therefore zone includes the nodes whose distance from the central node is at most $r$ hops. Each zone contains two types of nodes: peripheral nodes and interior nodes. Peripheral nodes are nodes whose minimum distance to the central node is exactly equal to the zone radius $r$ and interior nodes are nodes whose minimum distance to the central node is less than $r$ [8].

Zone Routing Protocol involves many components such as IARP, IERP and BRP, which only together provide the full routing benefit to ZRP. IntrA-zone Routing Protocol (IARP) is the locally proactive routing component of ZRP and IntEr-zone Routing Protocol (IERP) is the globally reactive routing component of ZRP. If a node sends a packet to another node then it should be checked whether the destination is outside the zone or within its local zone using information provided by IARP [9]. The packet can be routed proactively if its destination is within local zone and reactive routing is used if the destination is outside the zone. Due to the mobility of nodes, local neighborhood of a node may change rapidly. In IARP, to know about the topology of its routing zone, each node continuously maintains the routing information of all nodes within its routing zone. IARP can include any proactive routing protocol such as distance vector or link state routing for different routing zones and they are restricted within zones. In IERP [10], the routing process is divided into two phases: the route request phase and the route reply phase. In the route request phase, a route request packet is send by the source node to its peripheral nodes. If the receiver of a route request packet knows the destination, it responds by sending a route reply back to the source. Otherwise, it forwards the request packet to its neighbors. A node discards the route request if it receives the several copies of the same route request. In route reply phase, any node can send route reply packet if it provides a route to the destination. Each node along the path to the destination records the next-hop address in their routing table. The IERP takes the advantage of the local routing information provided by the IARP. It also handles route discovery if route request is initiated by peripheral nodes with by use of the Bordercast Resolution Protocol (BRP). BRP is used to direct route request generated by global reactive IERP to the peripheral nodes and utilizes the topology information provided by IARP to direct query request to the border of the zone. ZRP uses Neighbor Discovery Protocol (NDP) provided by the Media Access Control (MAC) layer to detect link failure and new neighbor nodes. NDP transmits HELLO beacons at regular intervals. The neighbor table is updated after receiving the HELLO beacon. If no beacon is received from a neighbor within a specified time, the entry of that neighbor is removed from the table. IARP provides this functionality of NDP, if NDP is not supported by MAC layer. 


\section{SIMULATION ENVIRONMENT}

We performed simulations on QualNet version 5.0 simulator and assumed two different scenarios for the performance evaluation of AODV, FSR and ZRP routing protocol. We have used two types of scenario in simulations:

1. Designed the network using random waypoint mobility model with different pause time.

2. Designed the network using random waypoint mobility model with variable number of nodes.

The Random waypoint model is a random-based mobility model designed to describe the movement pattern of mobile users, and how their location, velocity and acceleration change over time [15]. In random waypoint mobility model, the node selects a random position, moves towards it in a straight line at a constant speed that is randomly selected from a range, and pauses at that destination. The node repeats this, throughout the simulation.

The simulation parameters for scenariol and 2 are summarized in table 1 and table 2 respectively. Traffic sources for both networks are Constant Bit Rate (CBR).

Table 1: Simulation parameters for scenario 1

\begin{tabular}{|l|l|}
\hline Simulation parameter & Values \\
\hline No. of nodes & 50 \\
\hline Dimension of space & $1500 \mathrm{~m}$ X $1500 \mathrm{~m}$ \\
\hline Minimum velocity (v min) & $10 \mathrm{~m} / \mathrm{s}$ \\
\hline Maximum velocity (v max) & $20 \mathrm{~m} / \mathrm{s}$ \\
\hline Simulation Time & $300 \mathrm{sec}$ \\
\hline Item size & 512 bytes \\
\hline Source data pattern & 4 packets $/ \mathrm{sec}$ \\
\hline Node Placement Strategy & Random \\
\hline Pause time & $20 \mathrm{~s}, 40 \mathrm{~s}, 60 \mathrm{~s}, 80 \mathrm{~s}, 100 \mathrm{~s}$ \\
\hline No. of simulations & 15 \\
\hline
\end{tabular}

Table 2: Simulation parameters for scenario 2

\begin{tabular}{|l|l|}
\hline Simulation parameter & Values \\
\hline No. of nodes & $20,40,60,80,100$ \\
\hline Dimension of space & $1500 \mathrm{~m} \mathrm{X} \mathrm{1500m}$ \\
\hline Minimum velocity (v min) & $10 \mathrm{~m} / \mathrm{s}$ \\
\hline Maximum velocity (v max) & $20 \mathrm{~m} / \mathrm{s}$ \\
\hline Simulation Time & $300 \mathrm{sec}$ \\
\hline Item size & $512 \mathrm{bytes}$ \\
\hline Source data pattern & 4 packets/sec \\
\hline Node Placement Strategy & Random \\
\hline Pause time & $30 \mathrm{sec}$ \\
\hline No. of simulations & 15 \\
\hline
\end{tabular}

To evaluate the performance of routing protocols, we used four different quantitative metrics to compare the performance of AODV, FSR and ZRP routing protocols. They are Average endto-end delay, Packet delivery ratio, Throughput and Average Jitter.

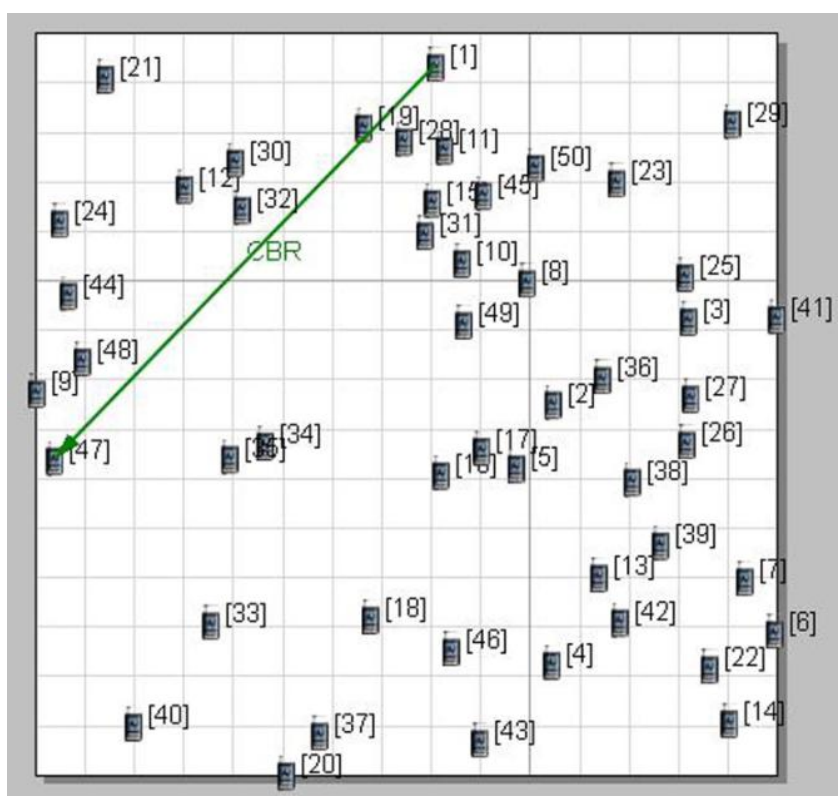

Figure 1: Snapshot of network in Qualnet5.0 Simulator

\section{SIMULATION RESULTS AND ANALYSIS}

\subsection{Average End-to-End Delay}

End-to-end delay indicates how long it a packet takes to travel from the CBR source to the application layer of the destination. [12]. This includes all possible delays caused by buffering during route discovery latency, queuing at the interface queue, retransmission delays at the MAC, propagation and transfer times. The average delay from the source to the destination's application layer is shown in figure 2 and figure 3 for scenario 1 and scenario 2 respectively. According to our simulation results, in scenario 1, best performance is shown by FSR having lowest end to end delay with a maximum delay of $.049 \mathrm{sec}$. With the increase in pause time average end to end delay first increases then decreases for AODV and FSR but in case of ZRP it is constant from pause time $60 \mathrm{~s}$ to $100 \mathrm{~s}$. But in scenario 2 , best performance is shown by ZRP having lowest end to end delay with a maximum delay of $.007 \mathrm{sec}$. The average end-to-end delay is always below from $0.065 \mathrm{~s}$ for all three protocols but in case of AODV it is very high initially and drastically decreases when pause time increases from 20 s to 40 s.

\subsection{Packet Delivery Ratio}

Packet delivery ratio is the fraction of packets sent by the application that are received by the receivers and is calculated by dividing the number of packets received by the destination through the number of packets originated by the application layer of the source. For better performance of a routing protocol, it should be better [13]. The packet delivery ratio is shown in 
figure 4 and figure 5 for scenario 1 and scenario 2 respectively. In scenario 1, AODV perform much better than FSR and ZRP as AODV delivers more than 60 percent of all CBR packets initiated by the source at different pause time while FSR and ZRP deliver only 25 percent of all CBR packets. In scenario 2 , AODV again perform much better than FSR and ZRP as AODV delivers more than 80 percent of all CBR packets initiated by the source at different pause time while FSR and ZRP deliver not more than 40 percent of all CBR packets.

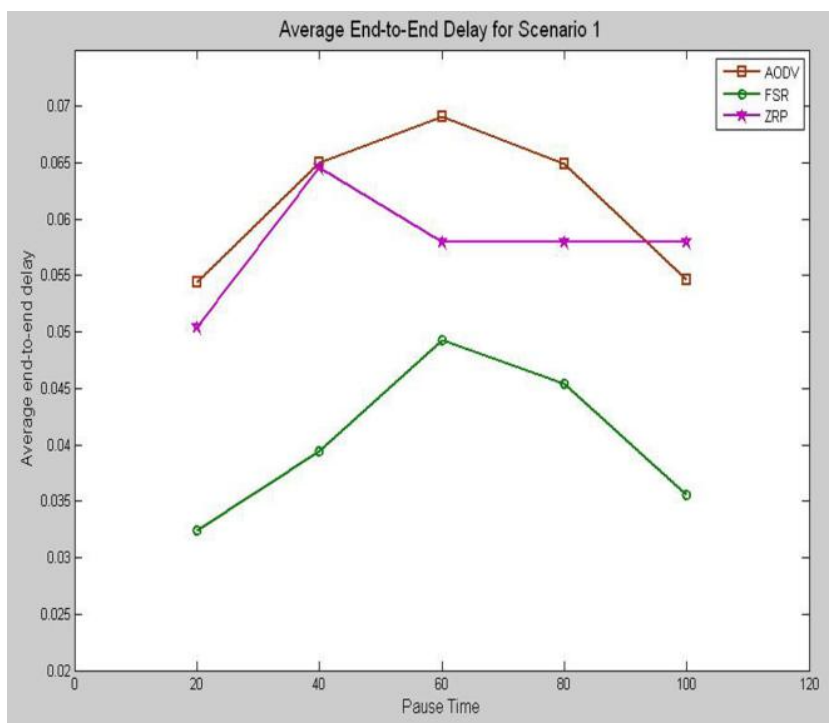

Figure 2: Average End to End Delay based on variable pause time

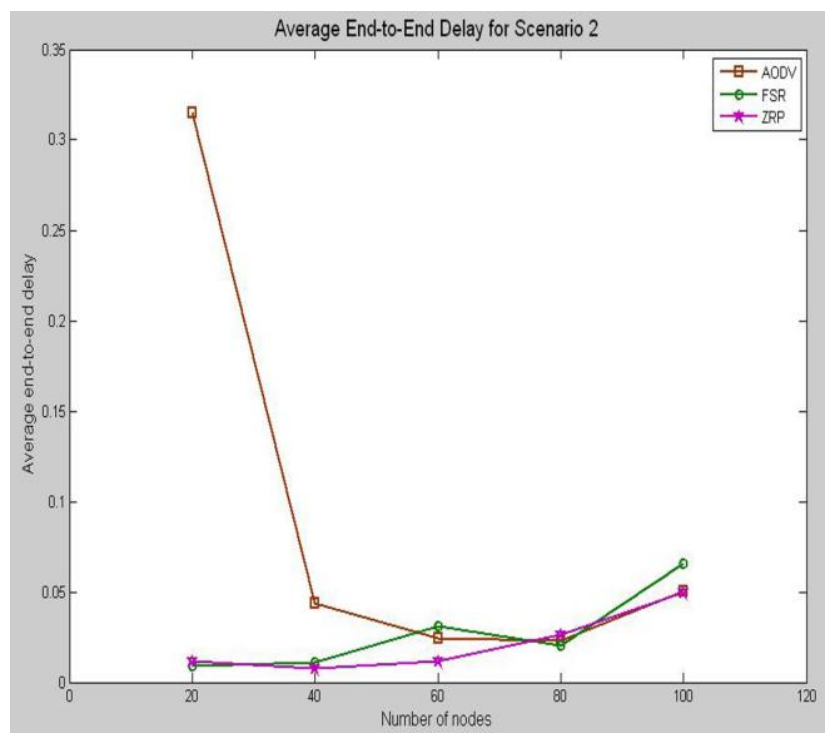

Figure 3: Average End to End delay based on variable number of nodes

\subsection{Throughput}

The throughput is defined as the total amount of data a receiver receives from the sender divided by the time it takes for the receiver to get the last packet. The throughput is measured in bits per second (bit/s or bps) [14]. The throughput is shown in figure 6 and figure 7 for scenario 1 and scenario 2 respectively. According to our simulation results, in both scenarios, best performance is shown by AODV as it delivers data packets at higher rate in comparison to FSR and ZRP.

\subsection{Average Jitter}

Jitter is the variation in the time between packets arriving, caused by network congestion, timing drift, or route changes. It should be less for a routing protocol to perform better. The average jitter is shown in figure 8 and figure 9 for scenario 1 and scenario 2 respectively. In AODV, there is more chance for jitter as source node initiate route discovery mechanism by broadcasting a route request packet to its neighbors. According to our simulation results, ZRP has less average jittering than AODV and FSR routing protocols in both scenarios.

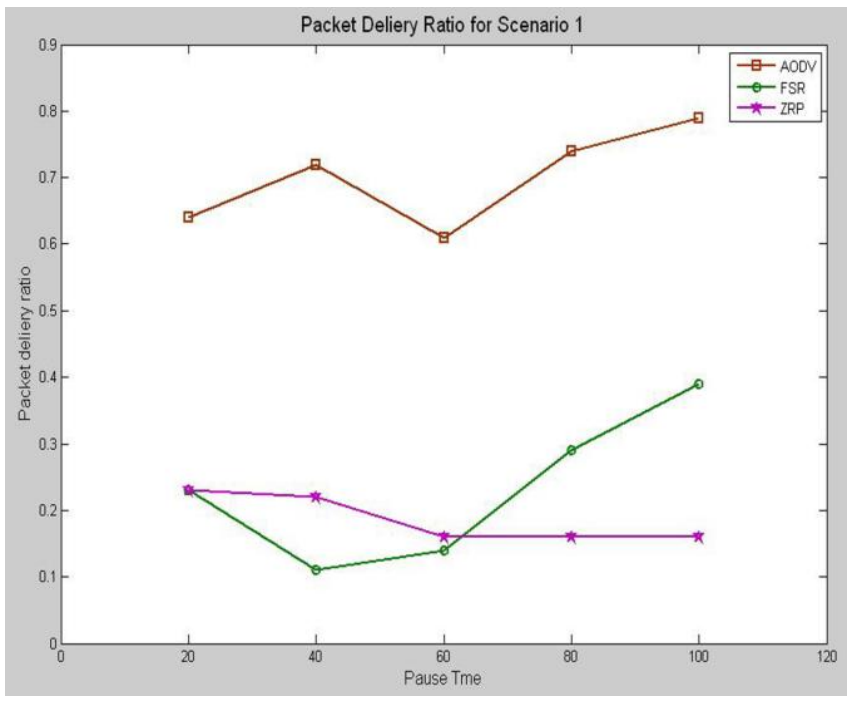

Figure 4: Packet Delivery Ratio based on variable pause time 


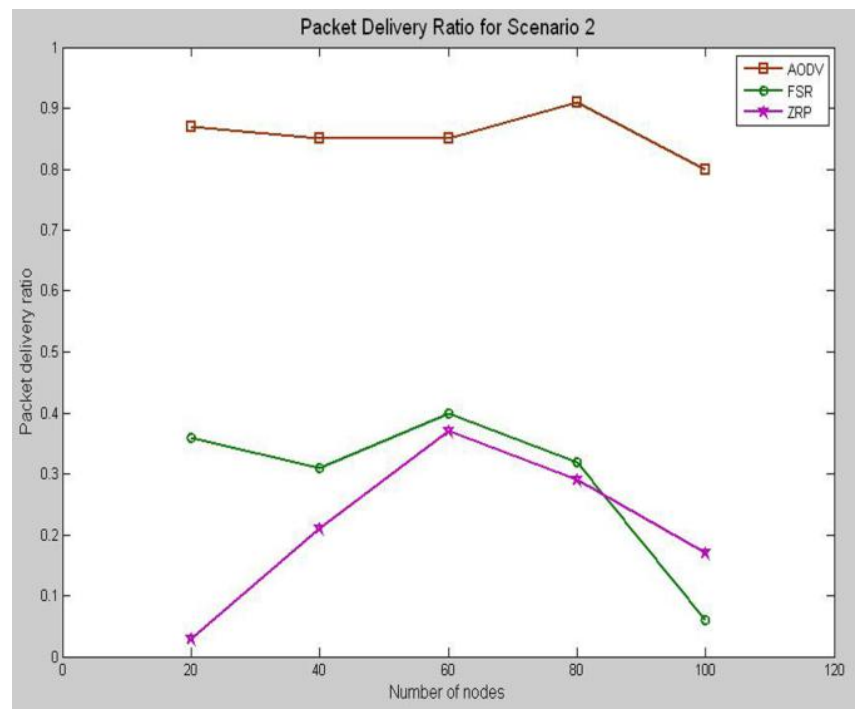

Figure 5: Packet Delivery Ratio based on variable number of nodes

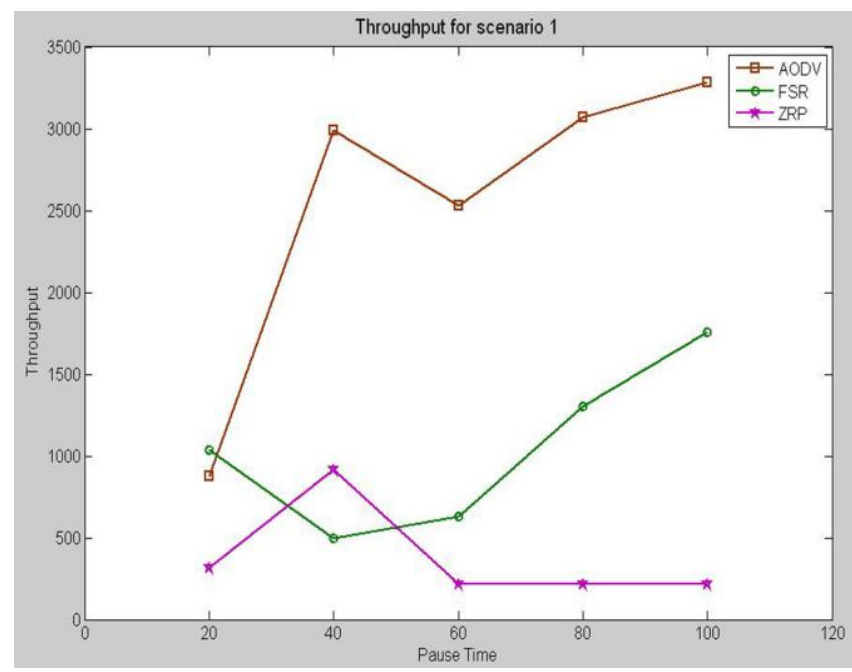

Figure 6: Throughput based on variable pause time

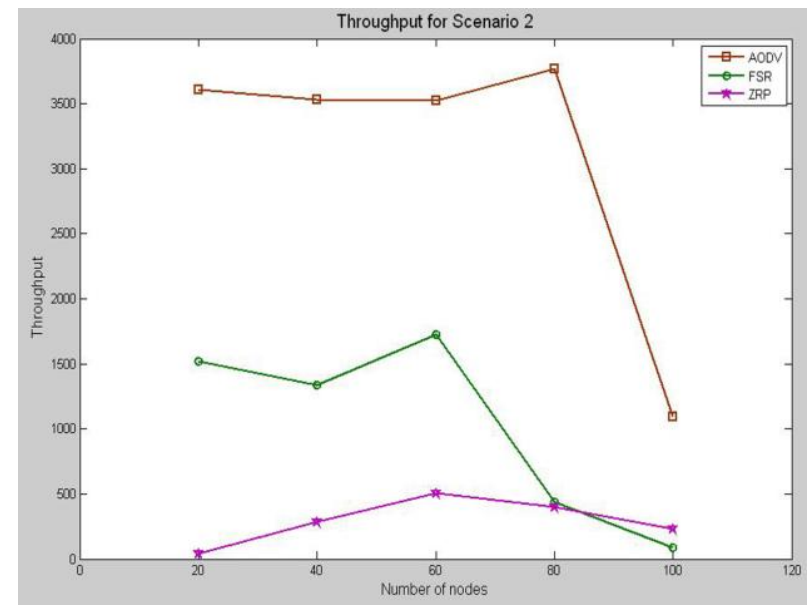

Figure 7: Throughput based on variable number of nodes

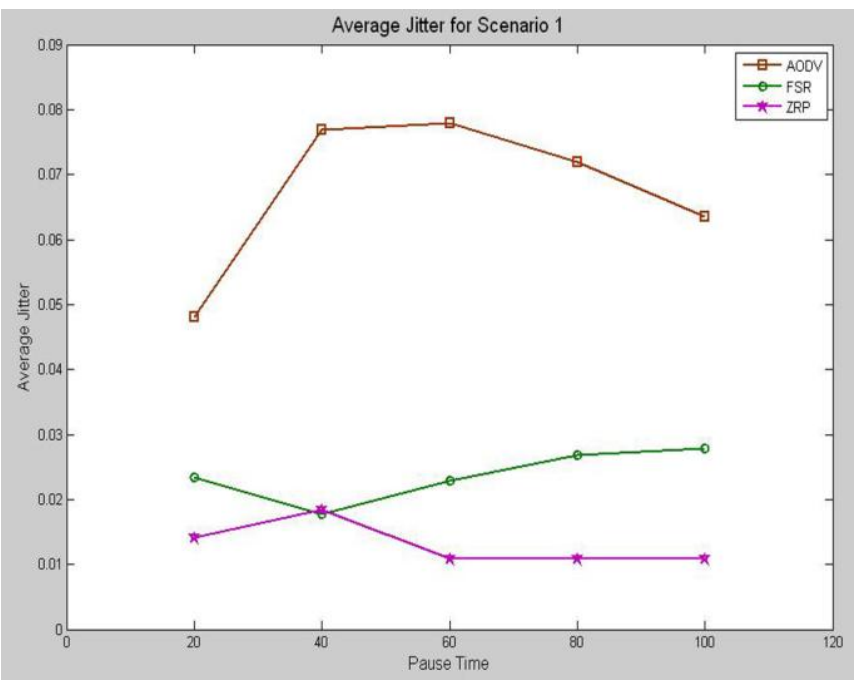

Figure 8: Average Jitter based on variable pause time

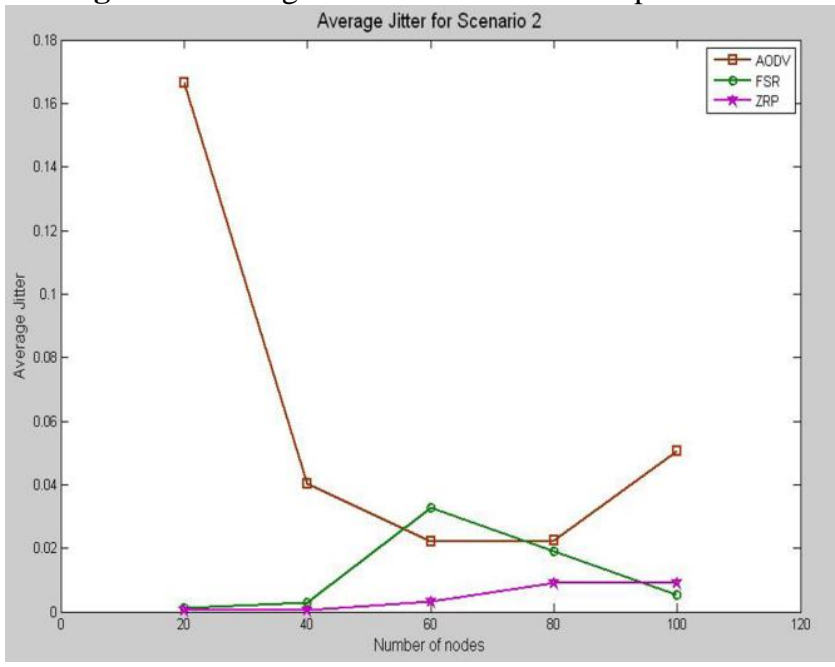

Figure 9: Average Jitter based on variable number of nodes

\section{CONCLUSIONS}

In this paper, a performance comparison of AODV, FSR, ZRP routing protocol for mobile Ad-hoc networks is presented as a function of pause time and as a function of number of node. Performance of AODV, FSR and ZRP routing protocol is evaluated with respect to four performance metrics such as average end to end delay, packet delivery ratio, throughput and average jitter. AODV shows best performance than FSR and ZRP in terms of packet delivery ratio and throughput. AODV delivers more than 60 percent of all CBR packets when network is presented as a function of pause time and delivers more than 80 percent of all CBR packets when network is presented as a number of nodes. According to our simulation results, FSR in scenario 1 and ZRP in scenario 2 show lowest end-to-end delay. In both scenarios, ZRP has less average jittering than AODV and FSR. Also in both scenarios, AODV performed the worst in case of average jitter and ZRP performed the worst in case of throughput. In future, different node placement strategy, more 
sources, additional metrics such as TTL based average hop count, routing overhead may be used.

\section{REFERENCES}

[1] The QualNet simulator www.scalable-networks.com.

[2] Elizabeth, Royer, Chai-Keong, Toh: "A Review of Current Routing Protocols for Ad Hoc Mobile Wireless Networks," IEEE Personal Communications, April 1999,

[3] Charles E. Perkins and Elizabeth M. Royer. "Ad hoc OnDemand Distance Vector Routing." Proceedings of the 2nd IEEE Workshop on Mobile Computing Systems and Applications, pp. 90-100, New Orleans, LA, February 1999.

[4] Chakeres, I.D.; Belding-Royer, E.M.; "AODV routing protocol implementation design," Proceedings of the 24th International Conference on Distributed Computing Systems Workshops (ICDCSW’04), pp. 698- 703, 23-24 March 2004

[5] Guangyu Pei; Gerla, M.; Tsu-Wei Chen; "Fisheye state routing: a routing scheme for ad hoc wireless networks," IEEE International Conference on Communications, 2000.

[6] Zone Routing Protocol by Nicklas Beijar, Networking laboratory, Helsinki University Of technology, Finland. Licentiate course on Telecommunications Technology, 2002

[7] Z. Haas and M. Pearlman. The performance of query control scheme for the zone routing protocol. ACM/IEEE Transactions on Networking, 9(4) pages 427-438, August 2001.

[8] Pearlman, Marc R., Haas, Zygmunt J.: Determining the Optimal Configuration for the Zone Routing Protocol, IEEE Journal on Selected Areas in Communications, Vol. 17, No. 8, August 1999

[9] Haas, Zygmunt J., Pearlman, Marc R., Samar, P.: Intrazone Routing Protocol (IARP), June 2001, IETF Internet Draft, draft-ietf-manet-iarp-01.txt
[10] Haas, Zygmunt J., Pearlman, Marc R., Samar, P.: Interzone Routing Protocol (IERP), June 2001, IETF Internet Draft, draft-ietf-manet-ierp-01.txt

[11] Haas, Zygmunt J., Pearlman, Marc R., Samar, P.: The Bordercast Resolution Protocol (BRP) for Ad Hoc Networks, June 2001, IETF Internet Draft, draft-ietf-manetbrp-01.txt

[12] David Oliver Jorg, "Performance Comparison of MANET Routing Protocols In Different Network Sizes", Computer Science Project, Institute of Computer Science and Applied Mathematics, Computer Networks and Distributed Systems (RVS), University of Berne, Switzerland, 2003.

[13] Jorjeta G.Jetcheva and David B. Johson, "A Performance Comparison of On-Demand Multicast Routing Protocols for Ad Hoc Networks," school of computer science, computer science department, Pittsburgh, December 15, 2004

[14] U. T. Nguyen and X. Xiong, "Rate-adaptive Multicast in Mobile Ad-hoc Networks," IEEE International Conference on Ad hocand Mobile Computing, Networking and Communications 2005 (WiMob 2005), Monreal, Canada, August 2005.

[15] Broch, J; Maltz DA, Johnson DB, Hu Y-C, and Jetcheva J. "A performance comparison of multi-hop wireless ad hoc network routing protocols". Proceedings of the Fourth Annual ACM/IEEE International Conference on Mobile Computing and Networking(Mobicom98), ACM, October 1998.

[16] QualNet5.0. Available. [Online]. http://www.scalablenetworks.com 